\title{
Manual Detorsion of Testicular Torsion - A Primary Care Intervention Procedure.
} Case Reports.

\section{E L Mugalo}

Moi University, department of medical Physiology and Moi Teaching and Referal Hospital, Department of Urology, Eldoret, Kenya.

Email: edwardmugalo@gmail.com.

Testicular torsion is one of the known acute urological emergencies that require prompt intervention. Salvage of the testis is only possible if derotation is performed within 6 hours of onset of symptoms. The objective of this paper is to report successful manual detorsion of the testes of patients with testicular torsion. Three cases with testicular torsion requiring emergency scrotal exploration underwent manual detorsion after sedation while waiting to be taken to theatre. Case 1, a 15 year old male diagnosed with right sided testicular torsion. Case 2, a 28 year old male with right testicular torsion. Case 3, a 15 year old male who presented with a 6 hour history of left testicular torsion. Attempting manual detortion in patients with acute testicular torsion can salvage the testis against loss.

Key Words: testicular torsion, manual detorsion, testicular survival, orchidopexy.

DOI: http://dx.doi.org/10.4314/ecajs.v21i3.21

\section{Introduction}

Testicular torsion is a known urological emergency that is characterized by twisting of the spermatic cord leading to acute pain and ischemia of the affected testes. It has a short window period within which salvage can be achieved. Common signs include swelling and redness of the scrotum (in light skinned people), nausea and vomiting, high riding testes, tender, swollen and firm with absent cremasteric reflex. A clear clinical diagnosis and early intervention is essential to avoid testicular loss. Approximately 1 : 4000 males younger than 25 years and $1: 160$ males by the age of 25 years are affected ${ }^{1,2}$. The "bell clapper" deformity is the underlying anatomical anomaly and it accounts for $90 \%$ of all cases (a). Acute scrotal pain in all prepubertal and young adult males could indicate testicular torsion until proven otherwise. It had been demonstrated both clinically ${ }^{3}, 4$ and experimentally 5 that testicular survival depends on the duration of ischemia. Testicular salvage rate is over $98 \%$ when diagnosis and detorsion occurs within 6 hours ${ }^{6}$. The affected testes can only be preserved by detorsion of the spermatic cord to restore perfusion. This can be achieved pre-operatively by manual detorsion or intra-operatively under direct vision.

\section{Case Reports}

We report three cases of successful reduction following detorsion, one with oral analgesia and the other with sedation and the third patient with Intramuscular analgesic injection. Scrotal exploration followed soon after and it confirmed successful manual detorsion. Bilateral orchidopexy was thereafter performed.

\section{Case 1}

A 15 year old male who developed scrotal pain and swelling at 2a.m. He was brought to casualty at 6 am, three (3) hours after the onset of pain, when the pain and swelling got worse. Physical examination revealed an enlarged right hemi scrotum. It was raised, tender and the testes assumed a transverse longitudinal axis. The tenderness was worsened by elevation of the scrotum, and cremasteric reflex was . Ultrasound performed in casualty revealed testicular torsion.

He was received in theatre $7 \frac{1}{2}$ hours after the onset of pain. Manual detorsion was attempted and successfully achieved after sedation for induction of general anesthesia.

When the scrotal sac was opened, the testes was found to be pink, area of torsion on the spermatic cord noted, bilateral orchidopexy was performed with non-absorbable silk2/0 suture. 


\section{Case 2}

A 28 year old man reported to our clinic with 4 hour history of sudden onset right scrotal pain, nausea and vomiting with no dysuria or urethral discharge. On examination he had an enlarged right scrotum, which was elevated with the testes lying transversely testes. The testicle was tender to touch, elevated with absent cremasteric reflex. The Patient initially declined surgery, insisting on U/S which confirmed absent Doppler flow in the right testes.

Manual detorsion was attempted by rotating the testes in anticlockwise direction, after giving oral analgesic, as we waited for the patient to consent for surgery. At surgery blood flow had already been reestablished in the right testes with the testes viable.

\section{Case 3}

A 15 year old male patient reported to accident and emergency with a 6 hour history of sudden onset left scrotal pain radiating to the lower abdomen. It was associated with nausea and vomiting, but no dysuria, fever or body chills. . A Doppler ultrasound done in another hospital before referral, confirmed absent color flow. On examination the left hemiscrotum was raised, tender to palpation. The testes had assumed a transverse orientation and the cremasteric reflex was absent. There was a palpable knot in the spermatic cord above the testes. An intramuscular injection of Tramadol 50mg stat was given in casualty and the patient got completely relived of pain. Manual detorsion was successfully performed in casualty and a repeat Doppler ultrasound revealed a swollen left testes with a normal Doppler color flow .The patient taken to the operating theater for scrotal exploration where the left testes was found to be viable. Bilateral orchidopexy using a 3/0 silk suture was performed.

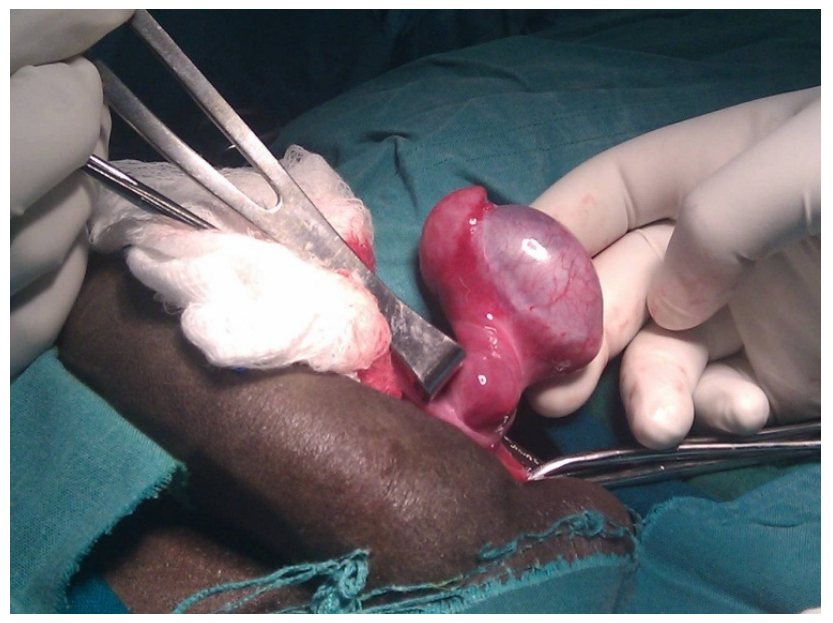

Figure 1. Salvaged Left Testes after Manual Distortion of Case 3.

\section{Discussion}

Testicular torsion is one of the known acute urological emergencies that require prompt intervention. Salvage is only possible if detorsion is performed within 6 hours of onset 6,7 .

Prolonged period of ischemia leads to loss of the affected testes. Most torsion of the spermatic cord is from lateral to medial. The preponderance to medial rotation ranges from $67 \%$ to $71 \%{ }^{8}$. Manual distortion has been reported since 1893 but is not widely practiced ${ }^{9}$. It provides an immediate noninvasive treatment for the problem. The affected tested is rotated away from the midline. Intravenous sedation with or without local analgesia (5mls of $2 \%$ lidocaine/xylocaine) infiltration around the spermatic cord near the external ring.

Manual detorsion aims at reversing the twisting through two planes. Due to the higher preponderance to medial rotation, the initial step at reduction involves rotation of the testes in a caudal cranial direction to release the locking mechanism. This is followed by a medial distortion of the spermatic cord. Sequential rotation by $180^{\circ}$ in one direction, initially lateral, due to higher predilection to medial rotation, then 


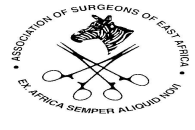

maintaining the twist after which it is followed by an attempt to a further $180^{\circ}$ or more. Increase in pain or increased resistance may indicate attempting distortion in the wrong direction.

Dramatic reduction in pain indicates success. Elective orchidpexy can be arranged if resumption of blood flow has been documented by Doppler ultrasound. Success rate has been reported at $26.5 \%$ in a metaanalysis ${ }^{10}$ while another put it at $80 \%{ }^{11}$. Lengthening of spermatic cord ,resolution of epididymal and spermatic cord edema, return to anatomical position and near or complete relief of testicular pain confirms successful detorsion ${ }^{\mathbf{1 2}}$. In a primary health facility where surgical intervention is not possible or is not quickly available, manual detorsion will greatly improve the salvage rate. Consequences of either delay in intervention or due to referral usually lead to testicular loss, and may attract litigation.

\section{Conclusion}

Testicular torsion is a urological emergency that requires prompt intervention to salvage the affected testes. Salvage rate is $98 \%$ when diagnosis and definitive treatment is performed within 6 hours. In view of the delays that are commonly experienced in our healthcare care system, knowledge of this condition and the procedure of manual detorsion will greatly improve the salvage rate in our set up. The health professional will also refer the patients early for definitive management where there is limitation of facilities for prompt surgical intervention.

\section{Acknowledgements}

To the Moi and Referral Hospital Management for giving permission to publish my experience in managing the three patients reported in this publication.

\section{References}

1. Erica Ringdal. MD and Lynn Teague MD.Testicular torsion. Am Fam Physician. 2006 Nov

15 ; 74(10):1739-1743.

2. Sinisi AA, Difinizio B, Lettieri $\mathrm{F}$ et al. Late gonadal function and autoimmunization in familial testicular torsion. Arch Andriol 1993; 30:147.

3. E.V. Cattolica, J. Karol, K, Rankin, and R, Klein. "High testicular salvage rate in torsion of the spermatic cord". The Journal of urology, vol; 128, no.1, pp.66-68, 1982.

4. E. Makela, T. Lahdes-Vasama, H.Rajakorpi, and S. Wikstrom. "spermatic cord block and manual reduction: Primary treatment for spermatic cord torsion". The Journal of Urology, vol: 132, no.5, pp, 921-923, 1984.

5. G. Smith. "Cellular changes from graded testicular ischemia". The Journal of urology, vol, 72, pp.355-362, 1955.

6. Paul Headway, John M Reynard. The six-hour rule for testis fixation in testicular torsion: Is it History? Journal of clinical urology March 2013 vol.6 no.2 84-88.

7. Makela E, Lahdes -vasama T, Rajakorpi H, Vikstrom S. a 19 year review of pediatric patients with acute scrotum. Scand J Surg. 2007; 96(1):62-6.

8. Session AE, Robinowitz R, Hulbert WG, Goldstein MM, Mevorah RA. Testicular torsion, direction, degree, duration and destination. J Urol 2003 Feb 169(2):663-5

9. Nash W, acute spermatic cord: reduction: immediate relief. British Medical Journal 1893; 1:P742.

10. Hwatrey CE. Assessment of acute scrotal symptoms and findings. A clinician's dilemma. Urol Clin North Am. 1908; 25:715-23.

11. Cornel EB, Karthaus HF. Manual derotation of the twisted spermatic cord. BJU Int. 1999; 83:672-4

12. Kiesling VJ Jr, Schroeder DE, Pauljev P, Hull J. spermatic cord block and manual reduction: Primary treatment for spermatic cord torsion. J urol 1984 Nov, 132(5):921-3. 